\title{
Encapsulation and Characterization of Fucoidan- Curcumin Nano Micelle for Anti-inflammatory Effects
}

\author{
Balaji Sadhasivam and Saraswathy Nachimuthu* \\ ${ }^{1}$ Department of Biotechnology, Kumaraguru College of Technology, \\ Coimbatore-641049, Tamilnadu, India.
}

\begin{abstract}
Curcumin is a potential bioactive compound used for many healthcare applications. Due to its hydrophobic nature therapeutic index is reduced many folds. Encapsulation of curcumin in a hydrophilic polymeric micelle enhances its bioavailability in biological systems. Fucoidan is a functional polysaccharide isolated from brown sea-weeds possess various health beneficial applications. In the present work, curcumin was encapsulated in fucoidan polymeric nano micelle (FCN). The optimal contact time for the encapsulation of curcumin was found to be 24 hours, average size of nano micelle formed $121+0.1 \mathrm{~nm}$ with 67\% curcumin encapsulation efficiency. Zeta potential was maximum of -47.4 which shows electro-kinetic potential in colloidal dispersions of Fucoidan Curcumin Nano Micelle (FCN). Physical Characterization of FCN was performed using FTIR spectroscopy and conjugation of fucoidan and curcumin was confirmed based on peak shift at wave number $2215 \mathrm{~cm}^{-1} ; 1639 \mathrm{~cm}-1 ; 1517 \mathrm{~cm}^{-1}$ and $1265 \mathrm{~cm}^{-1}$. Analysis of SEM, and TEM images were performed to know the nature of encapsulation of curcumin in polymeric micelle. Results obtained from in vitro assays such as antioxidant, anti-hemolytic and anti-inflammatory showed potential bioactive properties of FCNs.
\end{abstract}

KEY WORDS: ANTI-INFLAMMATORY EFFECTS, FUCOIDAN-CURCUMIN, NANO MICELLE, TEM, SEM, FTIR.

\section{INTRODUCTION}

Curcumin has been used from earlier days as a remedy for different diseases. It shows good antioxidant activity by scavenging the reactive oxygen species (ROS) and nitrogen species. It is also proved to be a potent anti-inflammatory compound by altering several metabolic pathways mainly involving downregulation of key enzymes like cyclooxygenase-2 (COX-2) and 5-lipooxygenase (Rahmani et.al., 2018). It also has antimicrobial, anti-cancer, anti-diabetic and anti-mutagenic activity (Rahmani et.al., 2018; Gonzalez-Ortega et.al.,

\section{ARTICLE INFORMATION}

${ }^{*}$ Corresponding Author: saraswathy.n.bt@kct.ac.in Received 9th Oct 2020 Accepted after revision 13th Dec 2020 Print ISSN: 0974-6455 Online ISSN: 2321-4007 CODEN: BBRCBA

Thomson Reuters ISI Web of Science Clarivate Analytics USA and Crossref Indexed Journal

\section{Clarivate
Analytics}

NAAS Journal Score 2020 (4.31) SJIF: 2020 (7.728)

A Society of Science and Nature Publication,

Bhopal India 2020. All rights reserved.

Online Contents Available at: http//www.bbrc.in/

Doi: http://dx.doi.org/10.21786/bbrc/13.11/17
2020). Curcumin has a low bioavailability as it is hydrophobic in nature. Under in vivo condition, curcumin is quickly metabolized leading to poor absorption (Wal et.al., 2019; Phan et.al., 2018).

As a result, only a small amount of active biomolecule is available in the systemic circulation to exert therapeutic effects. Bioavailability of the curcumin could be increased by making a complex with a suitable hydrophilic molecule. Many methods are developed to increase the bioavailability of curcumin (Chen et al., 2020; De Leo et al., 2018; Karthikeyan et al., 2020; Moballegh Nasery et al., 2020; Wong et al., 2019; Youssouf et al., 2019). One of the potential methods by conjugating curcumin to polymers namely polysaccharides to make it hydrophilic. Natural polysaccharides are potential drug carriers as they are hydrophilic, biodegradable, biocompatible and can be modified (Naveen and Shastri, 2019).

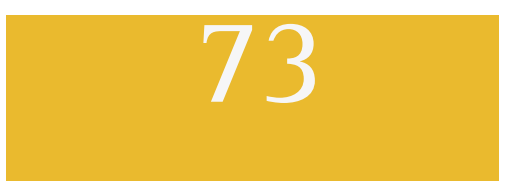


Polysaccharides modified with hydrophobic molecules can improve its self-assembly and enabling them to form micelles with increased oral and topical absorption (Naveen and Shastri, 2019). In micellar structure the hydrophobic regions face inside while the hydrophilic regions are exposed to the aqueous environment (Tran and Tran, 2019).

Fucoidan is a natural sulphated polysaccharide with high fructose content of around 60\% and hence it is widely used as health supplement for various applications. It is usually found in brown algae and is the most potent bioactive molecule. Studies have shown that fucoidan show good anti-inflammatory activity by inhibiting key enzymes which are responsible for inflammation. It is also a good antioxidant agent reducing the ROS and lipid peroxidation. Fucoidan is found to be a potential nanocarrier and has the ability to promote controlled drug delivery (Etman et.al., 2020). Studies have also shown that fucoidan to possess anti-inflammatory properties with suppression of enzymes like COX-2 (Manikandan et.al., 2020, Jeyawardena et.al., 2019). Conjugation of natural polysaccharides including pullulan and fucoidan with hydrophobic drugs and its use for cancer therapy has been previously studied (Grigoras, 2019, Guo et.al., 2020, Phan et.al., 2018). Fucoidan is a polysaccharide abundantly available in nature. Therefore, combining curcumin to fucoidan can improve the bioavailability of curcumin to increase its therapeutic effect.

In the current study, curcumin was encapsulated in fucoidan to form a Fucoidan Curcumin Nano micelle (FCN) and characterized using various techniques such as FTIR, SEM, TEM and zeta-potential. The bioactive properties of FCN were assessed by in vitro anti-oxidant, anti-haemolytic and anti-inflammatory assays.

\section{MATERIAL AND METHODS}

Materials: Fucoidan was extracted and purified from Sargassam longifolium a brown seaweed of Mandapam, Indian coastal region. Curcumin was procured from Sigma Aldrich, analytical grade ethanol and all other chemicals were used.

Preparation and Optimization Nano micelle: Fucoidancurcumin nanoparticles were synthesized by following desolvation method (Maghsoudi et al., 2017). The known concentration of curcumin was prepared in desolvating agent absolute ethanol and the polysaccharide solution of fucoidan $(1 \mathrm{mg} / \mathrm{mL})$ was dissolved in deionized water with $0.05 \%(\mathrm{v} / \mathrm{v})$ Tween 20 as an emulsifying agent. The curcumin-loaded fucoidan nanoparticles were prepared by adding desolvating agent dropwise to the fucoidan solution under continuous stirring. After different contact time, contents were rapidly centrifuged at 10,000 $\mathrm{rpm}$. The supernatant was centrifuged at $15,000 \mathrm{rpm}$ for 15 to 20 minutes to precipitate the nanoparticle. The free curcumin from precipitate was removed by absolute ethanol wash. The synthesized nanoparticles were re-suspended in deionized water The curcumin encapsulation in nano-micelle were calculated by following formula (Kamaraj et al., 2018).

Curcumin encapsulation $(\%)=\frac{\text { Amount of curcumin encapsulated }}{\text { Amount of Curcumin used }} \times 100$

Characterization: Particle size of nano micelle was measured by a nano-zetasizer (Malvern, UK). Fourier Transform Infra-Red Spectroscopy FT-IR ATR (IRAffinity $1 \mathrm{~S}$, SHIMADZU) was used to study the changes in the molecular level in spectral range from 4000 to $450 \mathrm{~cm}^{-1}$ with a resolution of $1.0 \mathrm{~cm}^{-1}$. Transmission electron micrograph (TEM) examination was performed in a Morgagni 268 D from FEI. A scanning electron micrograph (SEM) was recorded on a Jeol JSM 6390 microscope.

in vitro antioxidant assay: The free radical scavenging capacity of the nano micelle was evaluated by Diphenly picrylhydrazyl (DPPH) as free radical source (Chew et al., 2008). 20, 40, 60, 80 and $100 \mu \mathrm{L}$ of the compound was taken in a tube and made up the volume to $3 \mathrm{ml}$ with $0.1 \mathrm{mM}$ methanolic DPPH and incubated at $37^{\circ} \mathrm{C}$ for $20 \mathrm{~min}$. The absorbance was measured at $517 \mathrm{~nm}$ with methanolic DPPH solution as a negative control. The radical scavenging activity (RSA) was calculated as a percentage of DPPH discoloration using the equation:

DPPH Radical Scavenging Activity (\%) $=\frac{\text { OD of Control }- \text { OD of Test }}{\text { OD of Control }} \times 100$

in vitro anti-inflammatory activity-Human Red Blood Cells Membrane Stabilization assay: Anti-inflammatory assay was performed by investigating Fucoidan nano micelle FCN's human RBC membrane stabilization potential. The experiment was designed with modification of Bouhlali et al., (2016) method. The healthy volunteers with clear medical history from previous two weeks prior to experiment was selected. The whole blood was collected with equal volume of sterilized Alsever's solution (2\% dextrose, $0.80 \%$ sodium citrate, $0.05 \%$ citric acid and $0.42 \%$ sodium chloride). Centrifuged at 2500 rpm for 10 minutes. The plasma was removed and cell suspension layer washed with isosaline $(0.85 \%$ sodium chloride, pH7.2) and Centrifuged repeatedly until the supernatant was clear and colorless.

The HRBC component was resuspended in isosaline. The various concentrations of sample were prepared in distilled water. The reaction mixture equal volumes of sample, phosphate buffer $(0.15 \mathrm{M}, \mathrm{pH} 7.4)$, hyposaline $(0.36 \%$ sodium chloride) and HRBC suspension were incubated at $37^{\circ} \mathrm{C}$ for $30 \mathrm{~min}$. The content was centrifuged at $3000 \mathrm{rpm}$ for 20 minutes and then the supernatant was observed at $560 \mathrm{~nm}$ using spectrophotometer. Indomethacin was used as the reference drug. The percentage of membrane stabilization activity was calculated by using the formula, (Kumari et al., 2015).

Percentage Stabilization $=\frac{\text { OD of Control }- \text { OD of Test }}{\text { OD of Control }} \times 100$ 
in vitro anti-haemolytic assay: The haemolytic assay was performed by following method described by Mitra et al., (2015). Whenever RBC is subjected to irritating compound, cells will be lysed and haemoglobin will be released. The red blood cells were isolated from plasma and taken in Phosphate buffer saline. Different concentrations of samples were prepared in PBS. To

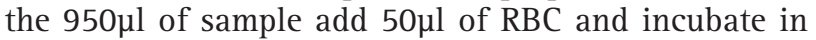
dark for 10 minutes. Centrifuge the content at 6000rpm for 10 minutes to separate the cell debris. Measure the absorbance at $540 \mathrm{~nm}$. Consider deionized water as positive control and PBS as negative control.

Percentage lysis $=\frac{\text { OD of Sample }- \text { OD of Positive Control }}{\text { OD of Negative Control }- \text { OD of Positive Control }} \times 100$

\section{RESULTS AND DISCUSSION}

Particle Size and Zeta Potential Analysis: The fucoidan based polymeric micelle loaded with curcumin was synthesized by desolvating method. Fucoidan is L-fuco-polysaccharide consisting of number of fucose monomers with $\alpha(1,4)$ glycosidic linkage. Curcumin is a low molecular weight drug molecule under desolvating condition gets entrapped inside the polymeric chain forming nano micellar structure. Thus, obtained fucoidan nano micelles FCN were analyzed for particle size, zeta potential, curcumin encapsulation efficiency and yield.

The optimum contact time of polymer and drug molecule is important to form nano micelle harboring shell made of polymeric ring encapsulating drug molecule in its core (Karthikeyan etl., 2020). The table 1, shows the size of the particle and zeta potential value for different contact time and it is evident that the contact time affects the size of the particle, zeta potential and curcumin encapsulation efficiency. Increasing the contact time, size of the nano micelle formed decreased and at 48 hours of contact time, nano micelle fraction showed particle size diameter 140+ $0.1 \mathrm{~nm}$ and -46.9 zeta potential values with maximum curcumin encapsulation efficiency of $74.01 \%$.

The higher positive and lower negative zeta potential value ensures the stability and monodispersity of the nano particle (Basniwal et al., 2011). The maximum measured zeta potential value is $-47.4 \mathrm{mV}$ at 24 hours of contact time. This negative charge density of FCN implies that the hydrophobic drug curcumin encapsulated possess hydrophilicity and it is expected that it could result in increased bioavailability than the free curcumin in the biological system. Hence is concluded that 48 hours of contact time is optimum for nano micelle preparation.

The surface topography of FCN nano micelle was examined using SEM as shown figure 1. The fucoidancurcumin formed a distinct structure without any aggregation and irregular clusters. The FCN nano micelle is in size of range $121 \mathrm{~nm}$ to $196 \mathrm{~nm}$. The figure 2(A $\mathrm{C}(\mathrm{B})$ represents the TEM image of fucoidan-curcumin nano micelle and figure 2 (C, D \&E) shows the core-shell properties of FCN analyzed using Image $\mathrm{J}$ software.
The figure 2 (D) shows the polymeric micelle having curcumin in its core and the figure 2(E) shows the fucoidan polymeric shell of FCN. Randomly 30 numbers of nano micelle structure were selected from processed TEM image of FCN (figure 2B) and analyzed for the core and shell diameter values. The Table 2 represents the average diameter and area of FCN. From the analysis, the average diameter of the core ranges from $84.7 \mathrm{~nm}$ to $160.9 \mathrm{~nm}$ and mean diameter is $115.6+18.3 \mathrm{~nm}$. The average diameter of the shell ranges from $186.4 \mathrm{~nm}$ to $288.2 \mathrm{~nm}$ and mean diameter is $222.8+23.5 \mathrm{~nm}$. This implies that fucoidan polymeric shell encapsulates the curcumin core as a nano micelle.

Table 1. Fucoidan curcumin Nano-Micelle at different
Contact Time
\begin{tabular}{l|c|c|c|c|}
\hline $\begin{array}{l}\text { Contact } \\
\text { Time in } \\
\text { (hrs) }\end{array}$ & $\begin{array}{c}\text { Size of } \\
\text { Particle } \\
\text { (nm + SD) }\end{array}$ & $\begin{array}{c}\text { Zeta } \\
\text { Potential }\end{array}$ & $\begin{array}{c}\text { Curcumin } \\
\text { encapsulation } \\
(\%+\text { SD) }\end{array}$ & $\begin{array}{c}\text { Yield } \\
(\%)\end{array}$ \\
\hline 4 & $196+0.2$ & -19.5 & $63.11+0.12$ & 45 \\
\hline 12 & $153+0.1$ & -41.8 & $64.61+0.11$ & 44 \\
\hline 24 & $121+0.1$ & -47.4 & $66.92+0.13$ & 49 \\
\hline 36 & $132+0.2$ & -46.3 & $68.17+0.11$ & 50 \\
\hline 48 & $140+0.1$ & -46.9 & $74.01+0.21$ & 50 \\
\hline
\end{tabular}

Figure 1: Scanning electron microscopic image of fucoidan-curcumin nano micelle
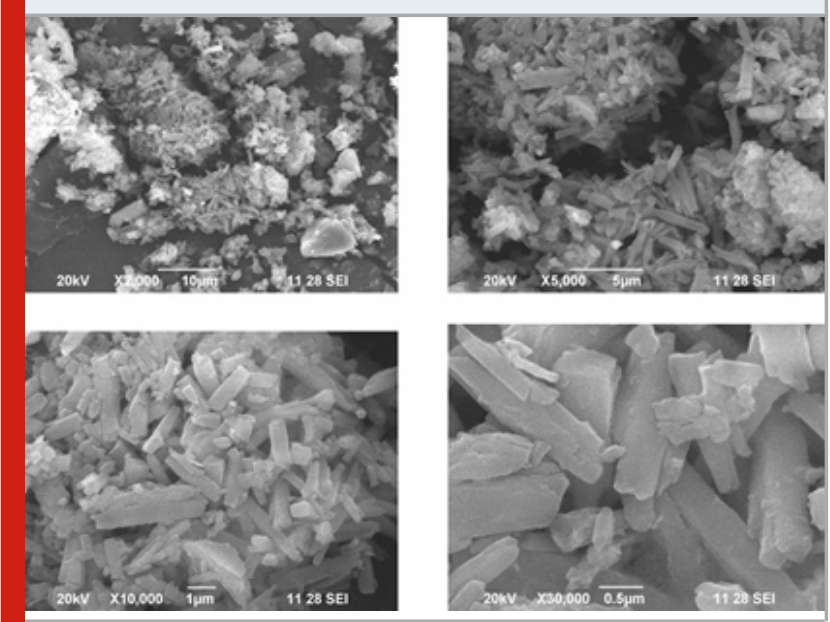

FT-IR Analysis: The Fourier Transform Infrared spectrum of fucoidan, curcumin and fucoidan curcumin nano micelle $(\mathrm{FCN})$ is represented in figure. 3. The FTIR spectrum of curcumin, fucoidan and FCN at 665,2100 and $3350 \mathrm{~cm}-1$ wave number represents the $-\mathrm{C}=\mathrm{C}-$ $\mathrm{H}: \mathrm{C}-\mathrm{H}$ bend of alkynes, $-\mathrm{C}=\mathrm{C}-$ stretch of alkynes and $\mathrm{O}-\mathrm{H}$ stretch vibrations of alcohols, phenols (Xu et al., 2014; Ye et al., 2013; Hifney et al., 2016). The fucoidan shows -C-N stretching of aromatic amines, $\mathrm{CH}$ stretching alkynes and $\mathrm{C}=\mathrm{C}$ stretching vibrations of aromatic rings at 1269, 1388 and $1546 \mathrm{~cm}^{-1}$ respectively (Xu et al., 2014; Marudhupandi et al., 2015). The curcumin exhibited the presence of C-0 Stretch alcohols carboxylic acids, 
esters, ethers, -C-N stretching of aromatic amines and CH stretching alkynes at 1024,1200 and $2943 \mathrm{~cm}^{-1}$ respectively ((Mohan et al., 2012; Kamaraj et al., 2018).

Figure 2: Transmission Electron Microscopic image of Fucoidan Curcumin Nano Micelle with 500nm scale. (A) TEM image of FCN before processing (B-E) TEM image of FCN after processing in ImageJ software. (C, D) shows the core of the FCN with average diameter of $115.6+18.3$ $\mathrm{nm}$. (E) shows the shell of FCN with average diameter of $222.8+23.5 \mathrm{~nm}$

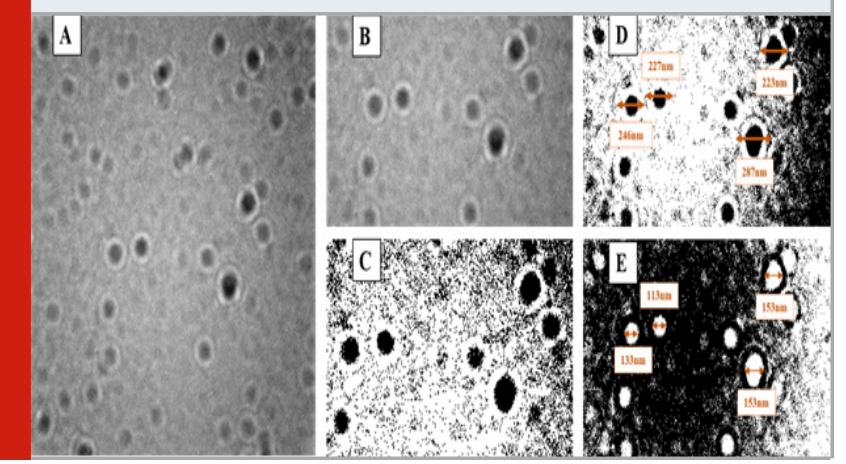

The wave number $1639 \mathrm{~cm}^{-1}$ and $2100 \mathrm{~cm}^{-1}$ shows the carbonyl $\mathrm{C}=0$ stretch of $\alpha, \beta$ unsaturated esters and presence of $-\mathrm{C}=\mathrm{C}$ - stretches of alkynes of fucoidan was retained in FCN. The FCN shows peak shift at $1269 \mathrm{~cm}^{-1}$ from $1100 \mathrm{~cm}-1$ of curcumin governing to -C0 stretching vibrations of aromatic rings and another peak shift was observed from $1469 \mathrm{~cm}^{-1}$ to $1549 \mathrm{~cm}^{-1}$ of curcumin having $-\mathrm{C}=\mathrm{C}$ stretching vibrations of aromatic rings. The $\mathrm{COOH}$ group, $\mathrm{CH}$ stretching of alkynes and C-C skeletal ring at 3311, 2943 and $1024 \mathrm{~cm}^{-1}$ of curcumin was not reflected in FCN indicating the encapsulation of curcumin in fucoidan polymeric micelle.

in vitro antioxidant assay: The DPPH Radical scavenging activity of curcumin, fucoidan and fucoidan-curcumin nano micelle was represented in figure 4. It was found that concentration of $100 \mu \mathrm{g} / \mathrm{ml}$ curcumin, fucoidan and fucoidan curcumin nano micelle (FCN) showed maximum scavenging activity of $98.9 \%, 85.8 \%$ and $97.1 \%$ respectively. The $\mathrm{IC}_{50}$ value of FCN was $40.7 \mu \mathrm{g} /$ ml which was similar to curcumin $39.1 \mu \mathrm{g} / \mathrm{ml}$ and lower than fucoidan $49.9 \mu \mathrm{g} / \mathrm{ml}$. It was evident that increasing the concentration DPPH scavenging percentage was also increased and curcumin encapsulated in FCN enhanced the antioxidant potential.

Table 2. Fucoidan Curcumin Nano micelle Core and Shell diameter analysis from ImageJ software

\begin{tabular}{|c|c|c|c|c|c|c|c|c|}
\hline \multirow[t]{2}{*}{ (in nm) } & \multicolumn{2}{|c|}{$\begin{array}{l}\text { Nano micelle Area } \\
\text { Range }\end{array}$} & \multicolumn{3}{|c|}{$\begin{array}{c}\text { Mean Nano micelle } \\
\text { Area }\end{array}$} & \multicolumn{2}{|c|}{$\begin{array}{l}\text { Nano micelle Diameter } \\
\text { Range micelle }\end{array}$} & \multirow{2}{*}{$\begin{array}{c}\text { Mean Nano } \\
\text { Diameter }\end{array}$} \\
\hline & Minimum & Maximum & Mean & SD & Minimum & Maximum & Mean & \\
\hline Shell & 1651.2 & 2512.7 & 1962.3 & 197.1 & 186.4 & 288.2 & 222.8 & 23.5 \\
\hline Core & 789.7 & 1435.8 & 1050.5 & 153.7 & 84.7 & 160.9 & 115.6 & 18.3 \\
\hline
\end{tabular}

Figure 3: FTIR Spectrum of Fucoidan Curcumin Nano Micelle (FCN), Curcumin and Fucoidan.

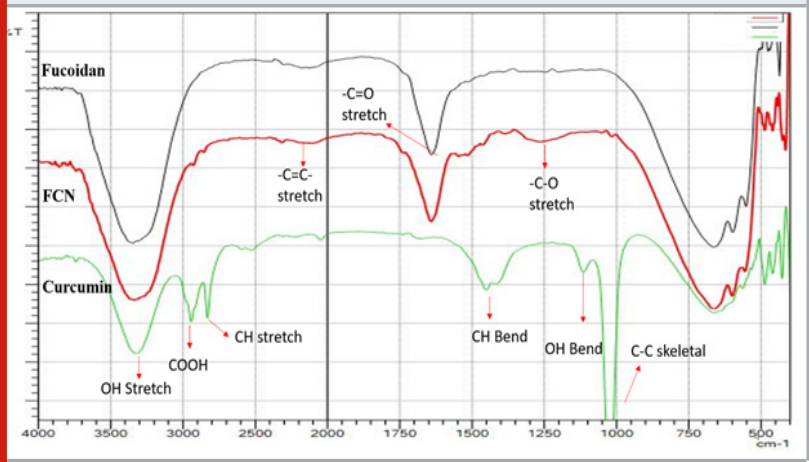

in vitro anti-inflammatory and anti haemolytic activity: The erythrocyte are predominantly available cells in human body and used extensively as biological model due to its morphological and physiological characteristics. During tissue injury or infection, the damaged cells and erythrocytes are exposed to the antigens leading to activation of immune responses. The stimuli activates inflammatory cells including macrophages, adipocytes, and the release of cytokines (TNF- $\alpha$, IL- 6 and IL-1 $\beta$ ), inflammatory C-reactive protein (CRP) and enzymes such as glutathione peroxidase (GPx), high-mobility group box 1 (HMGB1), cyclooxygenase (COX)-2, inducible nitric oxide synthase (iNOS), superoxide dismutase (SOD) and NADPH oxidase (NOX), which independently restores homeostasis and controls the pathogen growth.

Figure 4: DPPH scavenging activity of Curcumin, Fucoidan and Fucoidan Curcumin Nano micelle

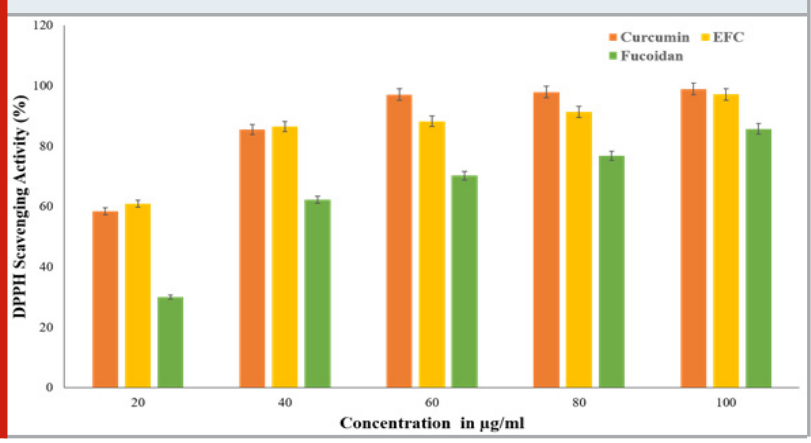

During abnormal inflammation, lysosomes releases excess enzymes into the cytosol which results in tissue damage. These inflammatory mediators target the erythrocyte membrane resulting in hemolysis and 
haemoglobin oxidation (Chen et al., 2018). Non-steroidal anti-inflammatory drugs (NSAIDs) inhibits the release of the inflammatory mediator's or by stabilizing the lysosomal membrane rendering the anti-inflammatory effect (Mounnissamy et al., 2007).

Figure 5: In vitro anti-inflammatory activity - hRBC membrane stabilization of Fucoidan Curcumin Nano micelle (FCN)

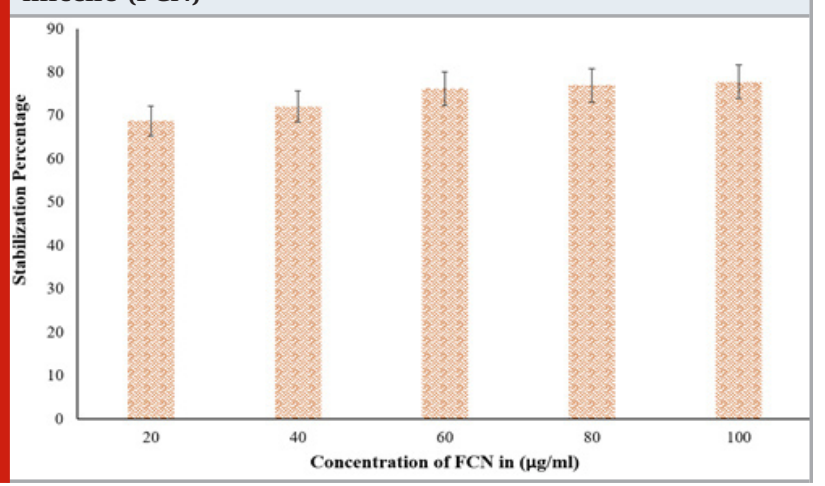

Figure 6: In vitro anti-haemolytic activity - Hemolytic inhibition percentage of Fucoidan Curcumin Nano micelle (FCN)

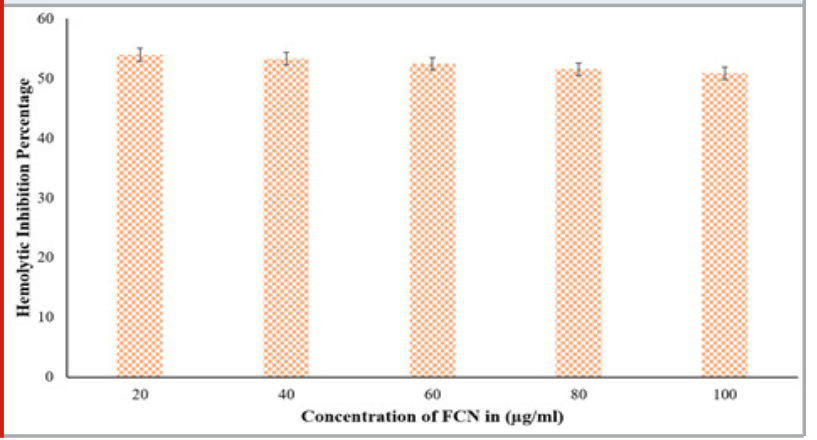

The human RBC stabilization activity of fucoidan curcumin nano micelle was represented in figure 5 . It was found increasing the concentration hRBC membrane stabilization was also increased. The maximum stabilization was observed at $100 \mu \mathrm{g} / \mathrm{ml}$ of FCN is $77 \%$ and $\mathrm{IC}_{50}$ was found to be $50.6 \mu \mathrm{g} / \mathrm{ml}$. The Anti hemolytic activity of fucoidan curcumin nano micelle was represented in figure 6. It was found that increasing the concentration fucoidan hemolytic inhibition was found to be the same at $54 \%$ and $\mathrm{IC}_{50}$ was found to be at $20.48 \mu \mathrm{g} / \mathrm{ml}$. Thus, the fucoidan curcumin nano micelle act as potential anti-inflammatory and blood compatible compound.

\section{CONCLUSION}

In this study curcumin was encapsulated in fucoidan to form a polymeric micelle (FCN). The successful encapsulation was confirmed by FTIR spectral analysis. SEM imaging showed no irregularities of nano clusters and no aggregates. TEM analysis showed the encapsulation of curcumin with curcumin in the core and fucoidan on the outer shell. Zeta potential showed the stability, monodispersity and hydrophilicity of FCN which signifies the potential for increased bioavailability of curcumin. The FCN showed to be a good antioxidant with 97.9\% activity. It also showed to be a potent hRBC stabilizing agent and with good anti-hemolytic potential with $\mathrm{IC}_{50}$ of $20.48 \mu \mathrm{g} / \mathrm{mL}$. The FCN has shown to possess several combined therapeutic benefits and with increased bioavailability serves as a potential dug candidate in various therapeutic applications.

\section{ACKNOWLEDGEMENTS}

This work was supported by Council of Scientific and Industrial Research, Ministry of Science and Technology, Government of India, India (Grant Number 08/677(0001)/ EMR-I) and Kumaraguru College of Technology, India. The structural elucidation of fucoidan were done at Council of Scientific and Industrial Research Institute Central Leather Research Institute, Chennai.

\section{REFERENCES}

Basniwal, R.K., Buttar, H.S., Jain, V.K. and Jain, N., (2011). Curcumin nanoparticles: preparation, characterization, and antimicrobial study. Journal of agricultural and food chemistry, 59(5), pp.2056-2061.

Bouhlali, E.T., Sellam, K., Bammou, M., Alem, C. and Filali-Zehzouti, Y., (2016). In vitro antioxidant and antiinflammatory properties of selected Moroccan medicinal plants. Journal of Applied Pharmaceutical Science, 6(5), pp.156-162.

Chen, L., Deng, H., Cui, H., Fang, J., Zuo, Z., Deng, J., Li, Y., Wang, X. and Zhao, L., (2018). Inflammatory responses and inflammation-associated diseases in organs. Oncotarget, 9(6), p.7204.

Chen, Y., Lu, Y., Lee, R.J. and Xiang, G., (2020). Nano Encapsulated Curcumin: And Its Potential for Biomedical Applications. International Journal of Nanomedicine, 15, p.3099-3120.

Chew, Y.L., Lim, Y.Y., Omar, M. and Khoo, K.S., (2008). Antioxidant activity of three edible seaweeds from two areas in South East Asia. LWT-Food Science and Technology, 41(6), pp.1067-1072.

De Leo, V., Milano, F., Mancini, E., Comparelli, R., Giotta, L., Nacci, A., Longobardi, F., Garbetta, A., Agostiano, A. and Catucci, L., (2018). Encapsulation of curcumin-loaded liposomes for colonic drug delivery in a pH-responsive polymer cluster using a pH-driven and organic solvent-free process. Molecules, 23(4), p.739. Etman, S.M., Elnaggar, Y.S. and Abdallah, O.Y., (2020). Fucoidan, a natural biopolymer in cancer combating: From edible algae to nanocarrier tailoring. International Journal of Biological Macromolecules, 147, pp.799808.

González-Ortega, L.A., Acosta-Osorio, A.A., GrubePagola, P., Palmeros-Exsome, C., Cano-Sarmiento, C., García-Varela, R. and García, H.S., (2020). Antiinflammatory Activity of Curcumin in Gel Carriers on Mice with Atrial Edema. Journal of Oleo Science, 69(2), pp.123-131. 
Grigoras, A.G., (2019). Drug delivery systems using pullulan, a biocompatible polysaccharide produced by fungal fermentation of starch. Environmental Chemistry Letters, pp.1-15.

Guo, C., Hou, X., Liu, Y., Zhang, Y., Xu, H., Zhao, F. and Chen, D., (2020). Novel Chinese Angelica Polysaccharide Biomimetic Nanomedicine to Curcumin Delivery for Hepatocellular Carcinoma Treatment and Immunomodulatory Effect. Phytomedicine, p.153356. Hifney, A.F., Fawzy, M.A., Abdel-Gawad, K.M. and Gomaa, M., (2016). Industrial optimization of fucoidan extraction from Sargassum sp. and its potential antioxidant and emulsifying activities. Food hydrocolloids, 54, pp.77-88.

Jayawardena, T.U., Fernando, I.S., Lee, W.W., Sanjeewa, K.A., Kim, H.S., Lee, D.S. and Jeon, Y.J., (2019). Isolation and purification of fucoidan fraction in Turbinaria ornata from the Maldives; Inflammation inhibitory potential under LPS stimulated conditions in in-vitro and in-vivo models. International journal of biological macromolecules, 131, pp.614-623.

Kamaraj, S., Palanisamy, U.M., Mohamed, M.S.B.K., Gangasalam, A., Maria, G.A. and Kandasamy, R., (2018). Curcumin drug delivery by vanillin-chitosan coated with calcium ferrite hybrid nanoparticles as carrier. European journal of pharmaceutical sciences, 116, pp.48-60.

Karthikeyan, A., Senthil, N. and Min, T., (2020). Nanocurcumin: A Promising Candidate for Therapeutic Applications. Frontiers in Pharmacology, 11.

Kumari, C.S., Yasmin, N., Hussain, M.R. and Babuselvam, M., (2015). Invitro anti-inflammatory and anti-arthritic property of Rhizopora mucronata leaves. Intern $\mathrm{J}$ Pharma Scie Res, 6, pp.482-485.

Maghsoudi, A., Yazdian, F., Shahmoradi, S., Ghaderi, L., Hemati, M. and Amoabediny, G., (2017). Curcuminloaded polysaccharide nanoparticles: Optimization and anticariogenic activity against Streptococcus mutans. Materials Science and Engineering: C, 75, pp.12591267.

Manikandan, R., Parimalanandhini, D., Mahalakshmi, K., Beulaja, M., Arumugam, M., Janarthanan, S., Palanisamy, S., You, S. and Prabhu, N.M., (2020). Studies on isolation, characterization of fucoidan from brown algae Turbinaria decurrens and evaluation of it's in vivo and in vitro anti-inflammatory activities. International Journal of Biological Macromolecules, 160, pp.12631276.

Marudhupandi, T., Kumar, T.T.A., Lakshmanasenthil, S., Suja, G. and Vinothkumar, T., (2015). In vitro anticancer activity of fucoidan from Turbinaria conoides against A549 cell lines. International journal of biological macromolecules, 72, pp.919-923.

Mitra, T., Manna, P.J., Raja, S.T.K., Gnanamani, A. and Kundu, P.P., (2015). Curcumin loaded nano graphene oxide reinforced fish scale collagen-a 3D scaffold biomaterial for wound healing applications. RSC Advances, 5(119), pp.98653-98665.

Moballegh Nasery, M., Abadi, B., Poormoghadam,
D., Zarrabi, A., Keyhanvar, P., Khanbabaei, H., Ashrafizadeh, M., Mohammadinejad, R., Tavakol, S. and Sethi, G., (2020). Curcumin Delivery Mediated by Bio-Based Nanoparticles: A Review. Molecules, 25(3), p.689.

Mohan, P.K., Sreelakshmi, G., Muraleedharan, C.V. and Joseph, R., (2012). Water soluble complexes of curcumin with cyclodextrins: Characterization by FT-Raman spectroscopy. Vibrational Spectroscopy, 62, pp.77-84. Mounnissamy, V.M., Kavimani, S., Balu, V. and Quine, S.D., (2007). Evaluation of Anti-inflammatory and Membrane stabilizing property of Ethanol Extract of Cansjera rheedii J. Gmelin (Opiliaceae). Iranian Journal of Pharmacology and Therapeutics, 6(2), pp.235-0.

Naveen, C. and Shastri, N.R., (2019). Polysaccharide nanomicelles as drug carriers. In Polysaccharide Carriers for Drug Delivery (pp. 339-363). Woodhead Publishing.

Phan, N.H., Ly, T.T., Pham, M.N., Luu, T.D., Vo, T.V., Tran, P.H. and Tran, T.T., (2018). A comparison of fucoidan conjugated to paclitaxel and curcumin for the dual delivery of cancer therapeutic agents. AntiCancer Agents in Medicinal Chemistry (Formerly Current Medicinal Chemistry-Anti-Cancer Agents), 18(9), pp.1349-1355.

Rahmani, A.H., Alsahli, M.A., Aly, S.M., Khan, M.A. and Aldebasi, Y.H., (2018). Role of curcumin in disease prevention and treatment. Advanced biomedical research, 7 .

Tran, T.T. and Tran, P.H., (2019). Nanoconjugation and encapsulation strategies for improving drug delivery and therapeutic efficacy of poorly water-soluble drugs. Pharmaceutics, 11(7), p.325.

Wal, P., Saraswat, N., Pal, R.S., Wal, A. and Chaubey, M., (2019). A Detailed Insight of the Anti-inflammatory Effects of Curcumin with the Assessment of Parameters, Sources of ROS and Associated Mechanisms. Open Medicine Journal, 6(1).

Wong, K.E., Ngai, S.C., Chan, K.G., Lee, L.H., Goh, B.H. and Chuah, L.H., (2019). Curcumin nanoformulations for colorectal cancer: a review. Frontiers in pharmacology, 10, p.152.

Xu, P., Wu, J., Zhang, Y., Chen, H. and Wang, Y., (2014). Physicochemical characterization of puerh tea polysaccharides and their antioxidant and $\alpha$-glycosidase inhibition. Journal of Functional Foods, 6, pp.545554.

Ye, H., Zhou, C., Li, W., Hu, B., Wang, X. and Zeng, X., (2013). Structural elucidation of polysaccharide fractions from brown seaweed Sargassum pallidum. Carbohydrate polymers, 97(2), pp.659-664.

Youssouf, L., Bhaw-Luximon, A., Diotel, N., Catan, A., Giraud, P., Gimié, F., Koshel, D., Casale, S., Bénard, S., Meneyrol, V. and Lallemand, L., (2019). Enhanced effects of curcumin encapsulated in polycaprolactone-grafted oligocarrageenan nanomicelles, a novel nanoparticle drug delivery system. Carbohydrate polymers, 217, pp.35-45. 